\title{
Ultrasonic Non-destructive Testing and in Situ Regulation of Residual Stress
}

\author{
Chunguang $\mathrm{Xu}^{1, \mathrm{a}^{*}}$, Haibing Tian ${ }^{2, \mathrm{~b}}$, Wentao Song ${ }^{3, \mathrm{c}}$ and Jianfeng Song ${ }^{4, \mathrm{~d}}$ \\ $1,2,3,4$ School of Mechanical Engineering, Beijing Institute of Technology, Beijing, China

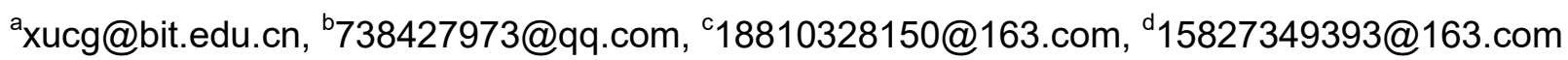

Keywords: Residual Stress, Ultrasonic, Non-Destructive Testing, Petroleum Pipeline

\begin{abstract}
Based on acoustoelasticity theory, the relationship between ultrasonic and stress in mechanical component is researched. Further, the principle, method and technology of exciting ultrasonic critically refracted longitudinal wave is analyzed. In order to ensure that test results are exact and realization of quantity traceability, the calibration technology of ultrasonic testing residual stress is studied. At last, the ultrasonic stress testing and calibration system is established. This technology now is widely used in testing residual stress in high-speed rail and petroleum pipeline.
\end{abstract}

\section{Introduction}

Residual stress greatly influences the service performance of practical engineering components, notably their strength, fatigue life, and dimensional stability. The fatigue life of metallic materials can be extended by the near-surface macroscopic compressive residual stresses that retard fatigue crack initiation and crack growth. Thus, it is important to know the magnitudes of these stresses, and to account for them during the design process. However, the "locked-in" character of residual stresses makes them challenging to measure, because there are no external loads that can be manipulated.

The engineering properties of materials and structural components, notably fatigue life, distortion, dimensional stability, corrosion resistance, and brittle fracture, can be considerably influenced by residual stresses ${ }^{[1]}$. Such effects usually lead to considerable outlays in the repair and restoration of parts, equipment, and structures. Accordingly, residual stress analysis is an essential stage in the design of parts and structural elements, and in the estimation of their reliability under actual service conditions $^{[2]}$.

Measurement of the actual stress in a structure or component is of great importance in all areas of engineering ${ }^{[3]}$. Non-destructive methods offer the obvious advantage of specimen preservation, and are particularly useful for production quality control and measurement of detailed calibrations on representative specimen material to valuable specimens. However, these methods commonly require provide the required computational data. A promising non-destructive method for subsurface stress measurement uses an acoustic transducer as an ultrasonic strain gauge. In this approach, ultrasonic measurements are taken before and after the specimen is subjected to stress ${ }^{[4]}$.

\section{Principle of residual stress testing}

The testing theory of acoustoelasticity

Acoustoelasticity theory is one of the main bases of ultrasonic stress testing. Acoustoelasticity theory is based on the finite deformation of continuum mechanics to study the relationship between the elastic solid stress state and the macroscopic elastic wave velocity. Based on the four basic assumptions of acoustoelasticity, the elastic wave equation (acoustoelasticity equation) in stress medium under initial coordinates can be obtained ${ }^{[5]}$. 


$$
\frac{\partial}{\partial X_{J}}\left[\left(\delta_{I K} t_{J L}^{i}+C_{I J K L}\right) \frac{\partial u_{K}}{\partial X_{L}}\right]=\rho^{i} \frac{\partial^{2} u_{I}}{\partial t^{2}}
$$

Where $\delta_{I K}$ is Kronecker delta function, $\rho^{i}$ is the density of the solid in the loading condition, $u_{I}$ is the dynamic displacement, $X_{J}$ is the particle position vector, $C_{I J K L}$ is the equivalent stiffness, which depends on the material constant and the initial displacement field and $t_{J L}^{i}$ is the Cauchy stress shown in the initial coordinates under the solid loading state.

In the case of homogeneous deformation and the solid is isotropic, Eq. (1) can be analytically expressed. Therefore, the equation for the ultrasonic propagation velocity and stress in solid can established in Cartesian coordinates ${ }^{[6]}$. For the longitudinal wave which propagates along the stress direction:

$$
\rho_{0} V^{2}=\lambda+2 \mu+\frac{\sigma}{3 \lambda+2 \mu}\left[\frac{\lambda+\mu}{\mu}(4 \lambda+10 \mu+4 m)+\lambda+2 l\right]
$$

In Eq. (2), $\lambda$ and ${ }^{\mu}$ are the Lame elastic constants; $l, m, n$ are the Murnaghan elastic constants; the elastic constants of different materials are shown in Table $1^{[7]} . \rho_{0}$ is the density of the solid before deformation; $\sigma$ is the stress applied in one direction (tensile stress is positive and compressive stress is negative); $V$ is the velocities of the longitudinal wave.

Table 1. Lame and Murnaghan constants of the materials, unit [GPa].

\begin{tabular}{llllll}
\hline Material & $\lambda$ & $\mu$ & $l$ & $m$ & $n$ \\
\hline Steel (1045) & 120 & 79 & -179 & -496 & -628 \\
Aluminium & 62 & 26 & -201 & -305 & -300 \\
$(6061)$ & & & & & \\
Copper (99.9\%) & 104 & 46 & $-542 \pm 30$ & $-372 \pm 5$ & $-401 \pm 5$ \\
\hline
\end{tabular}

The testing principle of ultrasonic LCR wave method

When a longitudinal wave propagates from a medium in which the wave velocity is slower to a medium in which the wave velocity is faster, according to the Snell law, there is an incidence angle that makes the refraction angle of the longitudinal wave equal to $90^{\circ}$. A longitudinal wave with a refraction angle equal to $90^{\circ}$ is called the critically refracted longitudinal wave $\left(\mathrm{L}_{\mathrm{CR}}\right.$ wave). The angle of incidence is the first critical angle.

We obtain the relationship between the longitudinal wave velocity that propagates along the stress direction and the stress, as shown in Eq. (2). In the actual detection, the distance between the transmitting and receiving transducer is fixed, and we can reflect the change of the sound velocity by calculating the change of the sound time and then determine the acoustic elastic effect. From Eq. (2), we can obtain the relationship between the stress variation and the changing time of sound propagation:

$$
d \sigma=K \cdot d t
$$




$$
K=\frac{-2 V_{0}(3 \lambda+2 \mu)}{\left(\frac{4 \lambda+10 \mu+4 m}{\mu}+\frac{2 l-3 \lambda-10 \mu-4 m}{\lambda+2 \mu}\right) L}
$$

Where, $K$ is stress coefficient of measured component, the unit is $\mathrm{MPa} / \mathrm{ns}$; $\Delta t$ is the time variation under the condition of stress; $L$ is the distance between the transmitting and receiving transducer, $V_{0}$ is the longitudinal wave velocity under the condition of zero stress.

\section{System of residual stress testing}

Schematic diagram of the testing system needs to meet the requirements are shown in Figure 1. It mainly includes specialized ultrasonic transducer, ultrasonic transceiver, temperature sensor and transmitter, automatic scan device, trigger and data collector, portable industrial control computer and corresponding algorithm software, calibration block, etc. The index of residual stress test system as follows: Detection range $\pm \sigma_{S}$ ( $\sigma_{S}$ is the yield strength of tested material); test resolution is $\pm 20 \mathrm{MPa}$; temperature range: $0^{\circ} \sim 40^{\circ}$; test depth: $0.5 \sim 10 \mathrm{~mm}$.

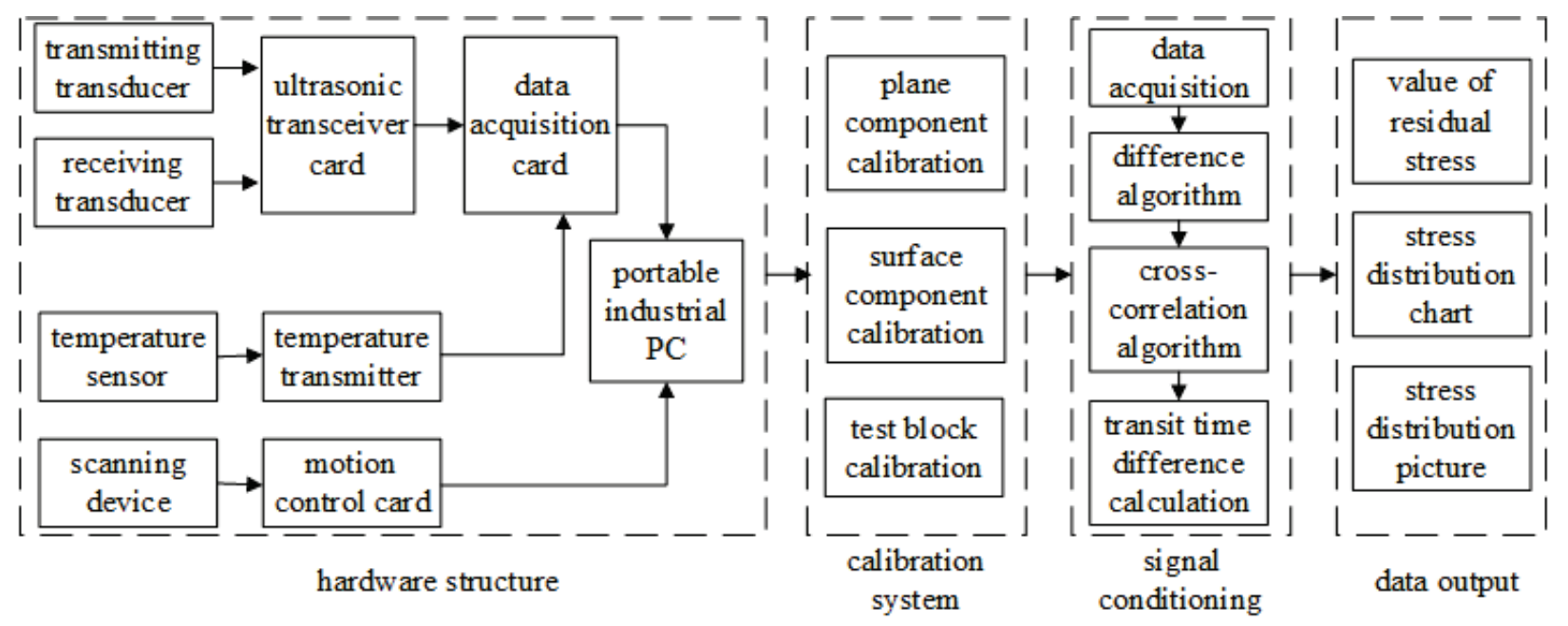

Fig. 1 Schematic diagram of residual stress testing system

\section{Experiment studies}

Residual stress testing for pipeline weld joint

Using the ultrasonic testing system to test residual stress of China's East-West pipeline, and evaluate the dangerous areas in pipeline, as shown in Fig. 2. The material of pipeline is X70 steel, and welding procedure is manual arc welding. We tested residual stress around straight weld joint in a section of pipeline. In order to verify the accuracy of the test results, a hydrostatic test has been carried out. From the Fig. 3, it is observed that the blasting place is consistent with the dangerous area evaluated by ultrasonic method. 


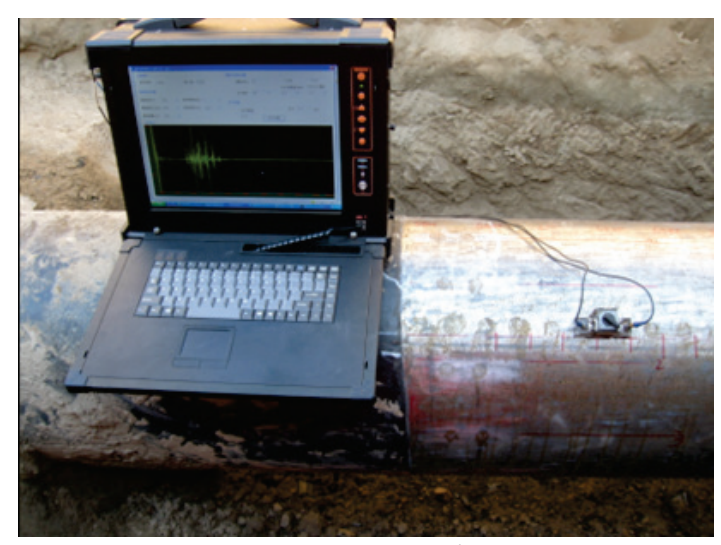

Fig. 2 Testing point location

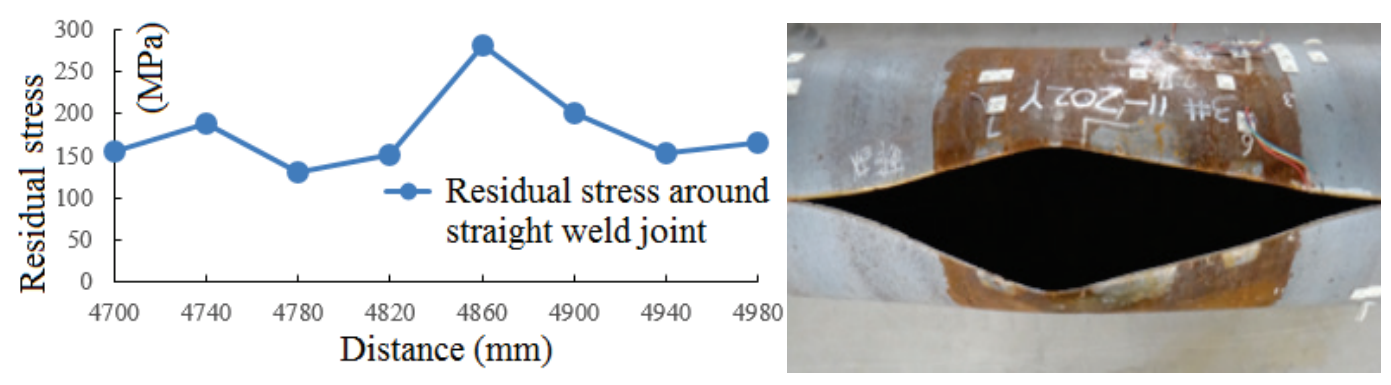

Fig. 3 Hydrostatic experimental verification

Residual stress distribution of steel pipe inner surface

The subject in this experiment is a steel pipe whose diameter is $105 \mathrm{~mm}$, wall thickness is $20 \mathrm{~mm}$. The material of pipe is 685 steel, and tested length is $4 \mathrm{~m}$. We test eight angles such as $0^{\circ}, 45^{\circ}, 90^{\circ}$, $135^{\circ}, 180^{\circ}, 225^{\circ}, 270^{\circ}$ and $315^{\circ}$. Every time, ultrasonic testing probe fixed in auto creeping mechanism moves along one of eight direction. Test residual stress while ultrasonic testing probe moves $40 \mathrm{~mm}$. Finally, the residual stress distribution of steel pipe inner surface is shown as in Fig. 4.

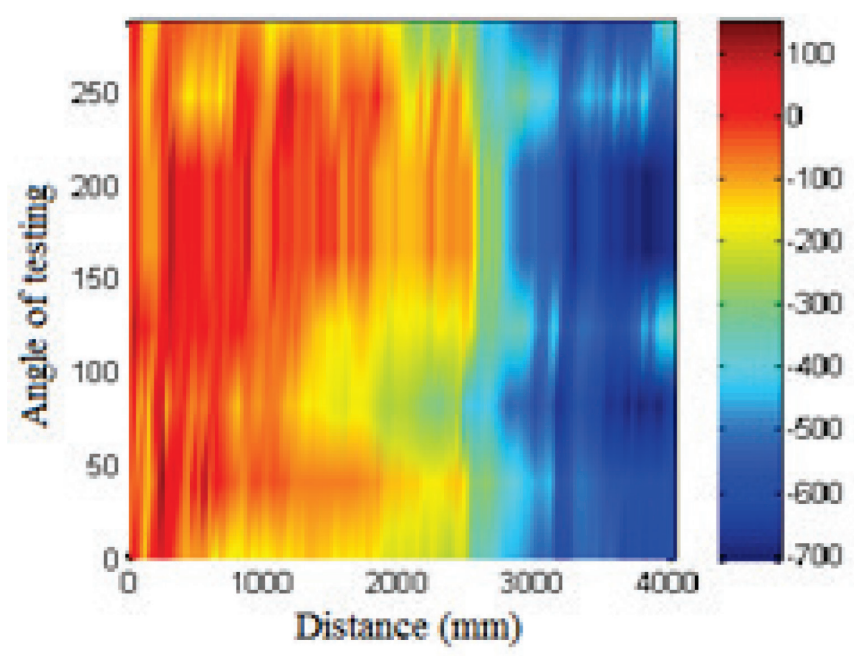

Fig. 4 Residual stress distribution of inner surface

Residual stress testing for rail

According to acoustoelasticity theory, we successfully accomplished the experiment of the residual stress of rail detection. 


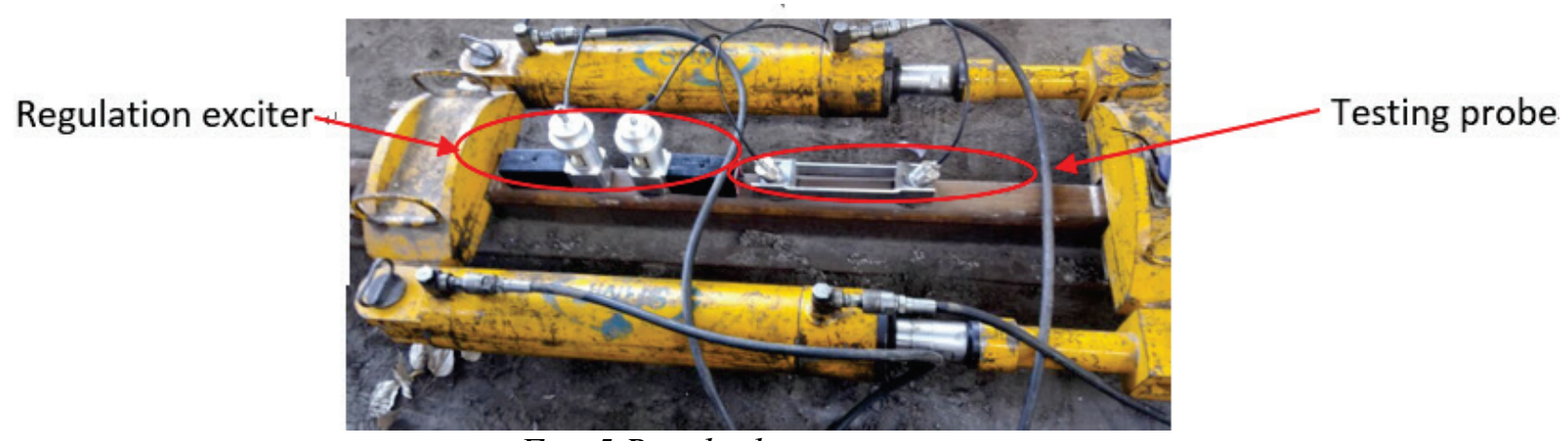

Fig. 5 Residual stress testing

The detection sensor used in this testing is shown in Fig. 5, which functions in the mode of pitch and catch. This detection sensor is composed of sound wedge and two ultrasonic transducer. Also, the distance of the inspiring and receiving ultrasonic transducer can be adjusted. Similarly, the angle of the ultrasonic transducer can also be adjusted. This will ensure the good coupling of the sensor and the sample and improve the detection accuracy.

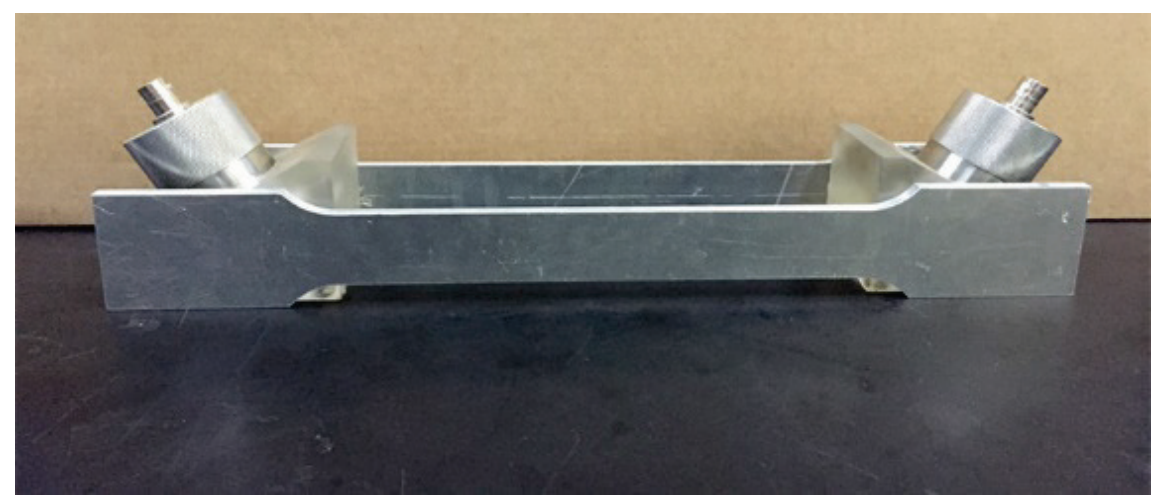

Fig. 6 Testing probe

As a rule, if the residual stress is in a state of compression, the rail is safe. Otherwise, it's in unsafe working condition. If the tensile stress is close to $1 / 3$ of the material yield strength, the rail is in dangerous condition. If the rail tensile stress is more than half of the yield strength, it is a very dangerous state. Thus, we need continuous monitoring when it works. If tensile stress is more than $2 / 3$ or $3 / 4$ of its yield strength. The rail is in a critical state of crack, we must stop its work.

\section{Conclusion}

(1) Based on acoustoelasticity theory, the principle of residual stress ultrasonic testing is analyzed. The stress sensitivity of different types of ultrasonic are compared, and it is obtained that longitudinal wave propagation along the stress direction is most sensitivity.

(2) According to theory of LCR wave, residual stress ultrasonic testing system for pipe and rail component is built.

(3) The residual stress in pipeline welding line, inner surface of steel pipe and rail are tested. Through hydrostatic experimental, the accuracy and practicability and universally application fields of the ultrasonic method can be verified.

\section{Acknowledgements:}

This work was sponsored by Natural Science Foundation of China (No. 51275042). 


\section{References}

[1] Totten, G. E., Howes, M. \& Inoue, T. Handbook of Residual Stress and Deformation of Steel. ASM International Publishers, USA, 2002 vol. 1, 417-444.

[2] Rossini, N. S., Dassisti, M., Benyounis, K. Y. \& Olabi, A. G.. Methods of measuring residual stresses in components. Mater. and Des. 2012, 35, 572-588. https://doi.org/10.1016/j.matdes.2011.08.022

[3] Gnaupel-Herold, T., Prask, H. J., Clark, A. V., Hehman, C. S. \& Nguyen, T. N. A comparison of neutron and ultrasonic determinations of residual stress. Meas. Sci. Technol. 2000, 11, 436-444. https://doi.org/10.1088/0957-0233/11/4/315

[4] Schajer, G. S. Relaxation methods for measuring residual stresses: techniques and opportunities. Experimental Mechanics. 2010, 50, 1117-1127. https://doi.org/10.1007/s11340-010-9386-7

[5] Bray, D. E., Junghans, P., Application of the Lcr Ultrasonic Technique for Evaluation of Postweld Heat Treatment in Steel Plates. NDT\&E International. 1995, 28, 235-242. https://doi.org/10.1016/0963-8695(95)00020-X

[6] Rose, J. L. Ultrasonic Waves in Solid Media. Cambridge: Cambridge University Press. 1999.

[7] Viktor, H. Structural and Residual Stress Analysis by Nondestructive Methods. Netherlands: Elsevier Press, 1997.

[8] Wentao Song, Chunguang Xu, Qinxue Pan, et al. Regulation of Residual Stress in Elastic Solid Component with High-energy Acoustic Field. AIP Conference Proceedings, 2013, 1511, 196-203. https://doi.org/10.1063/1.4789179 